\title{
EDITORIAL
}

\section{EGFRvIII: the promiscuous mutation}

\author{
Cell Death Discovery (2016) 2, 16049; \\ doi:10.1038/cddiscovery.2016.49; published online 4 July 2016
}

High-grade glioma (HGG) is the most common neoplasm of the brain and is almost universally lethal. ${ }^{1}$ One of the most frequently altered genes in HGG is the epidermal growth factor receptor (EGFR) gene. ${ }^{2}$ The most common alteration is amplification of wild-type (wt) EGFR, which is observed in around half of all HGG tumors. About half of these (about one-quarter of all HGG tumors) also contain a mutation known as EGFRvill, ${ }^{2}$ which is strongly tumorigenic. The signaling mechanisms underlying EGFRvill's tumorigenicity are not fully understood. Now, in a recent issue of Nature Neuroscience, Jahani-Asl et $a l^{3}{ }^{3}$ add to our knowledge of how EGFRvIll mediates its profound tumorigenicity.

Previous studies have shown that EGFRvIll is a gain-of-function mutation that arises from genomic deletion of exons $2-7 .{ }^{4}$ This deletion leads to a ligand-independent receptor, although the constitutive activity of this receptor is lower than that of ligandstimulated wtEGFR. ${ }^{4}$ Despite this sub-optimal activity, EGFRvill imparts a significant growth advantage to HGG cells, especially in vivo. Moreover, because of this low level of activation, EGFRvIll is not downregulated, unlike wtEGFR following activation by ligand. This leads to sustained signaling through EGFRvIll, ${ }^{5}$ which is critical to its tumorigenicity.

Nonetheless, this low level of sustained signaling through EGFRvIll is insufficient to explain its full tumorigenic activity, which promotes a diverse range of biological activities, including proliferation, survival and angiogenesis. ${ }^{4}$ There is some evidence of how EGFRvIll mediates its diverse range of biological effects. For example, we have shown that EGFRvIll signaling is amplified through interaction with a range of other receptor tyrosine kinases, especially c-Met. ${ }^{6}$ Indeed, EGFRvill forms a heterodimer with c-Met, leading to its direct activation and enhancing its response to the c-Met ligand hepatocyte growth factor. ${ }^{6}$ As a consequence, the co-targeting of EGFRvill and c-Met has synergistic anti-tumor activity in mouse xenograft models. ${ }^{6}$

In addition, EGFRvIll has been shown to promote HGG growth through paracrine mechanisms. Cells expressing EGFRvIll secrete large amounts of interleukin- 6 and leukemia inhibitory factor. ${ }^{7}$ These cytokines can activate signaling through gp130 in neighboring cells. Activated gp130, in turn, activates wtEGFR, even in the absence of EGFRvIII in these cells or of EGFR ligand, thereby enhancing HGG cell growth both in vitro and in xenograft models. ${ }^{7}$

The recent study by Jahani-Asl et $a .^{3}$ broadens our understanding of the tumorigenic capacity of EGFRvIll. The authors conducted global RNA sequencing (RNA-seq) on a series of HGG stem cell lines that express EGFRvill. Using the knowledge that EGFRvIll activates the transcription factor STAT3, they simultaneously performed RNA-seq on mouse astrocytes (one of the normal cells of origin for HGG) expressing EGFRvill in the presence or absence of Stat3. Finally, they also conducted chromatin immunoprecipitation sequencing on mouse astrocytes expressing both EGFRvIll and STAT3. Using this wealth of data, the authors were able to identify genes whose expression is driven specifically by the EGFRvIII-STAT3 pathway.

The primary 'hit' from this analysis was the receptor for the cytokine oncostatin $M$. This receptor, OSMR, was highly expressed in all tested HGG stem cell lines and in the EGFRvIII- expressing mouse astrocytes. Furthermore, STAT3 clearly bound to the Osmr gene promoter, and knockdown of Stat3 by lentivirus-mediated RNA interference resulted in a marked reduction in the level of Osmr mRNA, indicating that OSMR is probably a positive feed-forward signal in the EGFRvIII-STAT3 pathway (Figure 1).

Using classical biochemical techniques such as co-immunoprecipitation and newer imaging techniques such as proximity ligation assay, the authors also showed a direct interaction between the EGFRvIII and OSMR proteins. This interaction was dependent on the phosphorylation (activation) of EGFRvIll, as pharmacological inhibition of EGFRvIll with EGFR-directed tyrosine kinase inhibitors prevented the association of EGFRvIll and OSMR.

The canonical signaling pathway for OSMR involves binding its cognate ligand (OSM), direct interaction with its co-receptor (gp130) and subsequent activation of the JAK-STAT pathway. ${ }^{8}$ Surprisingly, the authors show that the interaction of EGFRvIll and OSMR and the subsequent signaling of the EGFRvIll-OSMR complex is independent of gp130. They go on to demonstrate a similar direct interaction between OSMR and wtEGFR; however, the formation of this receptor complex was dependent on OSM. One important follow-up question from these studies is whether the EGFRvIII-OSMR complex also directly activates JAK signaling and, as a corollary, whether this activation is augmented by the addition of exogenous OSM. If this pathway is activated, there is a readily available path into the clinic using FDA-approved JAK inhibitors such as ruxolitinib. ${ }^{9}$

EGFRvIll is known to contain a free cysteine that is not present in untruncated EGFR, and we have shown that this cysteine is critical for the activation of EGFRvIll and for its ability to transactivate other receptor tyrosine kinases such as c-Met. ${ }^{6}$ Uncovering the role of this cysteine in the EGFRvIII-OSMR complex may provide new mechanistic insight into how this complex is formed and subsequently signals.

Importantly, the authors also analyzed two HGG databases and showed that high levels of OSMR were significantly associated with poorer survival. Consistent with this, knockdown of OSMR in the HGG stem cell lines reduced their tumorigenicity in an intracranial model, and knockdown of Osmr in EGFRvIllexpressing mouse astrocytes robustly reduced their in vivo tumor growth by attenuating cell proliferation. Knockdown of Osmr in EGFRvill-expressing mouse astrocytes also had the surprising effect of significantly reducing EGFRvlll expression, which correlated with loss of phosphorylated STAT3. This raises the possibility that phospho-STAT3, driven by OSMR, may sustain EGFRvIll expression. This idea could be further explored in the human EGFRvIll-expressing HGG stem cell lines after OSMR knockdown. If replicated, the therapeutic targeting of OSMR could inhibit both EGFRvIII and STAT3 simultaneously. Taken together, these data suggest that targeting OSMR signaling in patients with EGFRvIll-positive HGG tumors would have therapeutic benefit.

This new study strongly supports the idea that EGFRvIII is a valid therapeutic target in HGG. Despite this, attempts to therapeutically target EGFR and EGFRvIll with single agents have largely failed in patients, ${ }^{10}$ even when the drug (e.g., gefitinib) reaches high enough concentrations within the tumor. ${ }^{11}$ This study raises the possibility that resistance to EGFR-targeted therapeutics could be mediated by EGFR- and/or EGFRvIll- 


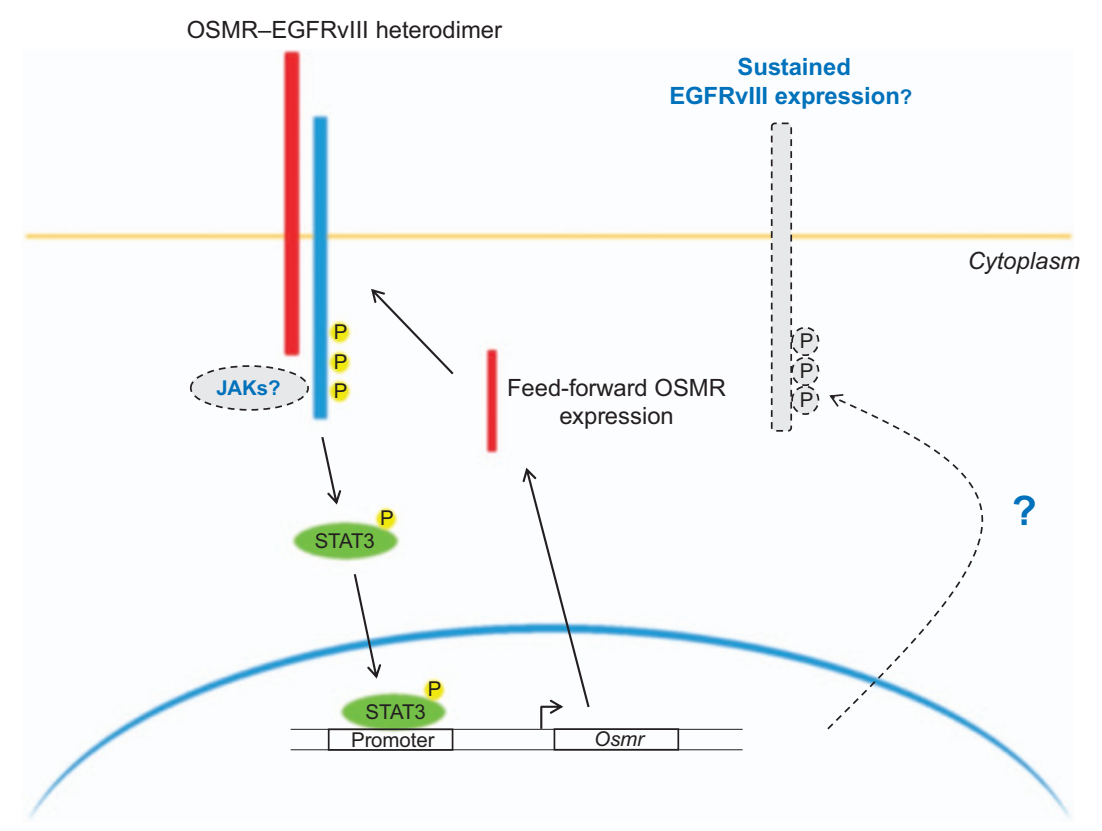

Nucleus

Figure 1. The EGFRvIII-OSMR heterodimer activates STAT3, initiating feed-forward expression of OSMR. EGFRvIII (blue) and OSMR (red) interact at the cell surface, leading to STAT3 (green) phosphorylation and phospho-STAT3 translocation to the nucleus. Nuclear phospho-STAT3 binds to the Osmr promoter and increases Osmr transcription. This results in feed-forward signaling through the EGFRvIll-OSMR heterodimer, which significantly increases HGG growth. Additional aspects of this model that could be explored in future are indicated by dashed lines. These include uncovering the role of JAK family kinases in activating STAT3 and determining whether phospho-STAT3 has a role in maintaining EGFRvIll expression. Yellow circles denote phospho-tyrosine.

independent OSMR signaling. This may involve OSM-OSMR autocrine loops or OSMR complexes with other receptor tyrosine kinases. Studies exploring this possibility are well warranted.

This is the first study to show how the previously described EGFRvIII-STAT3 signaling pathway ${ }^{12}$ contributes to the tumorigenicity of HGG cells and to identify OSMR as a therapeutic target. It also further highlights the promiscuous interactions between EGFRvIII and resident receptors in HGG cells. Understanding how these interactions function is vital for developing novel, efficacious therapies for HGG, a highly lethal cancer.

\section{COMPETING INTERESTS}

The authors declare no conflict of interest.

\section{ACKNOWLEDGEMENTS}

SAG and TGJ are members of the Brain Cancer Discovery Collaborative, which is supported by the Cure Brain Cancer Foundation. The Hudson Institute of Medical Research is supported by the Victorian Government's Operational Infrastructure Support Program. We thank Davina Dadley-Moore for editing the original manuscript.

Sameer A Greenall ${ }^{1,2}$ and Terrance G Johns ${ }^{1,2}$ ${ }^{1}$ Centre for Cancer Research, Hudson Institute of Medical Research, Clayton, Victoria 3168, Australia and

\author{
${ }^{2}$ Monash University, Clayton, Victoria 3800, Australia \\ E-mail: terry.johns@hudson.org.au
}

\section{REFERENCES}

1 Cloughesy TF, Cavenee WK, Mischel PS. Annu Rev Pathol 2014; 9: 1-25.

2 Brennan CW et al. Cell 2013; 155: 462-477.

3 Jahani-Asl A et al. Nat Neurosci 2016; 19: 798-806.

4 Gan HK, Cvrljevic AN, Johns TG. FEBS J 2013; 280: 5350-5370.

5 Schmidt MH et al. Proc Natl Acad Sci USA 2003; 100: 6505-6510.

6 Greenall SA et al. Oncogene 2015; 34: 5277-5287.

7 Inda MM et al. Genes Dev 2010; 24: 1731-1745.

8 Haan C et al. Biochem Pharmacol 2006; 72: 1538-1546.

9 Geyer HL, Mesa RA. Blood 2014; 124: 3529-3537.

10 Reardon DA, Wen PY, Mellinghoff IK. Neuro-oncology 2014; 16 (Suppl 8): viii7-13.

11 Hegi ME et al. Mol Cancer Ther 2011; 10: 1102-1112.

12 Fan QW et al. Cancer Cell 2013; 24: 438-449.

cC) (i) This work is licensed under a Creative Commons Attribution 4.0 International License. The images or other third party material in this article are included in the article's Creative Commons license, unless indicated otherwise in the credit line; if the material is not included under the Creative Commons license, users will need to obtain permission from the license holder to reproduce the material. To view a copy of this license, visit http://creativecommons.org/licenses/by/4.0/

(c) The Author(s) 2016 